\title{
NECESSARY CONDITIONS FOR ISOMORPHISM OF LIE ALGEBRAS OF BLOCK
}

\author{
BY
}

\author{
JOHN B. JACOBS
}

ABSTRACT. Two algebras of Block, $\mathcal{L}(G, \delta, f)$ and $\mathcal{L}\left(G^{\prime}, \delta^{\prime}, f^{\prime}\right)$, are isomorphic only if $m(G)=m\left(G^{\prime}\right)$. This is not sufficient for isomorphism.

Let $\mathcal{L}$ be a simple finite-dimensional Lie algebra over $\Phi$, an algebraically closed field of prime characteristic $p$. Simplicity allows the identification $x \leftrightarrow$ ad $x$ for each $x \in \mathfrak{L}$. (That $\mathfrak{L}$ be centerless is sufficient for the identification.). Then if $\mathscr{D}(\mathcal{Q})$ denotes the derivation algebra of $\mathcal{Q}$ we have $\mathfrak{L}(=\operatorname{ad} \mathscr{L}) \subset \mathcal{D}(\mathscr{Q})$. For each $x \in \mathscr{L},(\operatorname{ad} x)^{p}$ is a derivation of $\mathscr{L}$ and, if $(\operatorname{ad} \mathscr{L}) p^{k}$ is the vector space spanned by $\left\{(\operatorname{ad} x) p^{k} \mid x \in \mathcal{L}\right\}$, then $\mathscr{R}(\mathscr{I})=$ ad $\mathscr{L}$ $+(\operatorname{ad} \mathscr{L})^{p}+(\operatorname{ad} \mathscr{L}) p^{2}+\cdots$ is a subalgebra of $\mathscr{D}(\mathscr{Q})$ which is restricted. We will call $\mathscr{R}(\mathscr{Q})$ the restricted algebra of $\mathscr{L}$. If $\mathscr{I}$ is restricted, then ad $\mathscr{L}=$ $\Re(£)$, or under the identification, $\mathscr{L}=\mathscr{R}(£)$. Thus, for any arbitrary center-

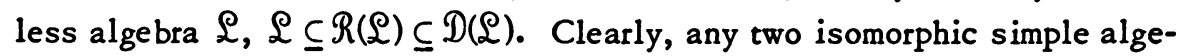

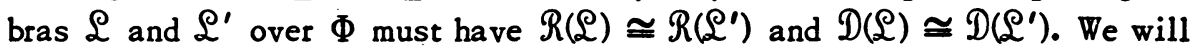
use this relationship to determine isomorphism conditions upon the algebras of Block.

Let $G$ be an elementary abelian $p$-group written as a direct summand of elementary abelian $p$-groups, $G=G_{0} \oplus G_{1} \oplus \ldots: \oplus G_{m}$. Let $\Phi$ be an algebraically closed field of characteristic $p>3$. For each $i=0,1, \ldots, m$ define $f: G \times G \rightarrow \Phi$ such that $\left.f\right|_{G_{i}}=f_{i}: G_{i} \times G_{i} \rightarrow \Phi$ is a skew-symmetric, nondegenerate biadditive form. Then $f=f_{0}+f_{1}+\cdots+f_{m}$. For each $i=1$, $\ldots, m$, assume that there exist additive functions $g_{i}, h_{i}: G_{i} \rightarrow \Phi$ such that $f_{i}(\alpha, \beta)=g_{i}(\alpha) h_{i}(\beta)-g_{i}(\beta) h_{i}(\alpha)$. Pick $\delta_{i} \in G_{i}$ for which $g_{i}\left(\delta_{i}\right)=0$, and set $\delta=\delta_{1}+\cdots+\delta_{m}$. Define $\mathcal{L}(G, \delta, f)$ to be the Lie algebra over $\Phi$ with basis $\left\{u_{\alpha} \mid \alpha \in G, \alpha \neq 0,-\delta\right\}$ where multiplication is given by

$$
u_{a} u_{\beta}=\sum_{i=0}^{m} f_{i}\left(a_{i}, \beta_{i}\right) u_{a+\beta-\delta_{i}} \text {. }
$$

Received by the editors January 17, 1974.

AMS (MOS) subject classification (1970). Primary 17B 40.

Key words and phrases. Algebras of Block, restricted algebra, isomorphism. 
Here $\alpha_{i}$ and $\beta_{i}$ denote the $i$ th components of $\alpha$ and $\beta$, respectively, in $G$ and $\delta_{0}$ is assumed to be zero. $\mathcal{L}(G, \delta, f)$ is then a simple algebra over $\Phi$ called an algebra of Block.

The derivations of the algebras of Block have been completely determined in [1]. As they will be utilized later, a brief description follows.

Since $G$ is an elementary abelian $p$-group it is an $n$-dimensional vector space over $\Phi_{p}$ (the prime subfield of $\Phi=G F(p)$ ), each of the $G_{i}$ 's being a subspace of dimension, say, $n_{i}$ Pick a basis $\left\{\sigma_{01}, \sigma_{02}, \ldots, \sigma_{0 n_{0}}\right\}$ for $G_{0}$ and $\left\{\sigma_{i 1}, \ldots, \sigma_{i n_{i}-1}, \delta_{i}\right\}$ for $G_{i}, i=1, \ldots, m$, such that $f\left(\sigma_{i 1}, \delta_{i}\right)=$ $f_{i}\left(\sigma_{i 1}, \delta_{i}\right) \neq 0$. Such is possible since $f$ is nondegenerate. For each $\alpha \in G$, write $\alpha=\Sigma_{i, j} s_{i j}(\alpha) \sigma_{i j}+\Sigma_{i} s_{i}(\alpha) \delta_{i}$. The coefficients $s_{i j}(\alpha), s_{i}(\alpha)$ of the $\sigma_{i j}$ 's and $\delta_{i}^{\prime}$ 's are unique since the $\sigma_{i j}$ 's and $\delta_{i}$ 's form a basis of $G$. The derivations of $\mathcal{L}(G, \delta, f)$ are linear combinations (over $\Phi$ ) of the elements in the following sets:

(i) $R=\left\{\operatorname{ad} u_{a} \mid a \in G, a \neq 0\right\}$ (ad $u_{-\delta}$ is included although not an element of $\mathcal{L}(G, \delta, f))$.

(ii) $S=\left\{D\left(\sigma_{k 1},-\delta_{k}\right), D\left(\delta_{k},-\delta_{k}\right) \mid k=1, \ldots, m\right\}$ where $u_{a} D\left(\gamma_{k},-\delta_{k}\right)=$ $f\left(\alpha, \gamma_{k}\right) u_{\alpha-\delta_{k}}$ for $\gamma_{k}$ in $G_{k}$.

(iii) $T=\left\{D\left(\sigma_{0 k}, 0\right), D\left(\sigma_{i j}, 0\right) \mid k=1, \ldots, n_{0} ; i=1, \ldots, m ; j=2, \ldots\right.$, $\left.n_{i}-1\right\}$ where $G_{0} \neq\{0\}$ and $T=\left\{D(\delta, 0), D\left(\sigma_{i j}, 0\right) \mid i=1, \ldots, m ; j=2, \ldots\right.$, $\left.n_{i}-1\right\}$ when $G_{0}=\{0\} ;$ where $u_{\alpha} D\left(\sigma_{i j}, 0\right)=s_{i j}(\alpha) u_{\alpha}$ and

$$
u_{a} D(\delta, 0)=\left(-1+\sum_{i} s_{i}(\alpha)\right) u_{\alpha^{\circ}}
$$

$\left(D\left(\sigma_{i 1}, 0\right)\right.$ is a linear combination of ad $u_{\delta_{i}}$ and the remaining $D\left(\sigma_{i j}, 0\right)$ 's.).

The set $S$ is, of course, empty when $m=0$. The dimension of $\mathscr{L}(G, \delta, f)$ is $n p^{n}-1$ for $m=0$ and $n p^{n}-2$ for $m>0$, and it follows that the dimension of its derivation algebra, $\mathcal{D}(\mathcal{Q}(G, \delta, f))$, is

(i) $n p^{n}+n-1$ when $G=G_{0}$ or when $G_{0} \neq 0$ and $m>0$.

(ii) $n p^{n}+n$ when $G_{0}=0$.

From the dimensions of the derivation algebras and their derived algebras, Block concludes in [1, Theorem 14, Corollary 1] that necessary conditions for two algebras $\mathcal{L}(G, \delta, f)$ and $\mathcal{L}\left(G^{\prime}, \delta^{\prime}, f^{\prime}\right)$ to be isomorphic are that either $G_{0}=0, G_{0}^{\prime}=0$, and $m(G)=m\left(G^{\prime}\right)$; or $G_{0} \neq 0 \neq G_{0}^{\prime}$ and $\min \{2, m(G)\}$ $=\min \left\{2, m\left(G^{\prime}\right)\right\}$. By considering the restricted algebra of $\mathscr{L}(G, \delta, f)$ we will show that it is necessary that $m(G)=m\left(G^{\prime}\right)$ for isomorphism and that, indeed, this is not sufficient.

For $u_{\alpha}, u_{\beta} \in \mathscr{L}(G, \delta, f)$ it is easily shown by induction on $m$ that 


$$
u_{\alpha}\left(\operatorname{ad} u_{\beta}\right)^{p}=\sum_{i=0}^{m} f\left(\alpha_{i}, \beta_{i}\right) f\left(\alpha_{i}-\delta_{i}, \beta_{i}\right) \cdots f\left(\alpha_{i}-(p-1) \delta_{i}, \beta_{i}\right) u_{\alpha}
$$

The following lemma then shows that

$$
u_{\alpha}\left(\operatorname{ad} u_{\beta}\right)^{p}=\sum_{i=0}^{m}\left\{f\left(\alpha_{i}, \beta_{i}\right)^{p}-f\left(\alpha_{i}, \beta_{i}\right) f\left(\delta_{i}, \beta_{i}\right)^{p-1}\right\} u_{\alpha^{*}}
$$

Lemma 1. Let $a, b \in \Phi$, char $\Phi=p>0$. Then

$$
a(a-b)(a-2 b) \cdots(a-(p-1) b)=a^{p}-a b^{p-1} .
$$

Proof. The polynomial $x^{p}-x b^{p-1}$ has roots $i b$ for $i=0, \ldots, p-1$. Hence, $x^{p}-x b^{p-1}=\Pi_{i=0}^{p-1}(x-i b)$. Substituting $a$ for $x$ yields the desired result.

It is evident that

$$
\begin{aligned}
u_{\alpha}\left(\operatorname{ad} u_{\beta}\right)^{p^{2}} & =\left\{\sum_{i=0}^{m} f\left(\alpha_{i}, \beta_{i}\right)^{p}-f\left(\alpha_{i}, \beta_{i}\right) f\left(\delta_{i}, \beta_{i}\right)^{p-1}\right\}^{p} u_{a} \\
& =\sum_{i=0}^{m}\left\{f\left(\alpha_{i}, \beta_{i}\right)^{p^{2}}-f\left(\alpha_{i}, \beta_{i}\right)^{p} f\left(\delta_{i}, \beta_{i}\right)^{p(p-1)}\right\} u_{\alpha},
\end{aligned}
$$

and more generally that

$$
u_{a}\left(\operatorname{ad} u_{\beta}\right)^{p^{k}}=\sum_{i=0}^{m}\left\{f\left(\alpha_{i}, \beta_{i}\right)^{p^{k}}-f\left(\alpha_{i}, \beta_{i}\right)^{p^{k-1}} f\left(\delta_{i}, \beta_{i}\right)^{p^{k-1}(p-1)}\right\} u_{a} .
$$

Suppose that $a=\Sigma_{i=0}^{m} a_{i}=\sum_{i=1}^{m}\left(\Sigma_{j=1}^{n_{i}-1} s_{i j}(\alpha) \sigma_{i j}+s_{i}(\alpha) \delta_{i}\right)+\Sigma_{j=1}^{n_{0}} s_{0 j}(\alpha) \sigma_{0 j}$. Then

$$
\begin{aligned}
\sum_{i=0}^{m}\left\{f\left(\alpha_{i}, \beta_{i}\right) p^{k}-f\left(\alpha_{i}, \beta_{i}\right)^{p^{k-1}} f\left(\delta_{i}, \beta_{i}\right)^{p k-1}(p-1)\right\} \\
=\sum_{i=0}^{m}\left(\sum_{j=1}^{q_{i}} s_{i j}(\alpha)\left\{f\left(\sigma_{i j}, \beta_{i}\right)^{p^{k}}-f\left(\sigma_{i j}, \beta_{i}\right)^{p^{k-1}} f\left(\delta_{i}, \beta_{i}\right)^{p^{k-1}(p-1)}\right\}\right. \\
\left.+s_{i}(\alpha)\left\{f\left(\delta_{i}, \beta_{i}\right)^{p^{k}}-f\left(\delta_{i}, \beta_{i}\right)^{p^{k-1}} f\left(\delta_{i}, \beta_{i}\right)^{p^{k-1}(p-1)}\right\}\right),
\end{aligned}
$$

or

$$
\operatorname{ad} u_{\beta}^{p^{k}}=\sum_{i=0}^{m}\left(\sum _ { j = 1 } ^ { q _ { i } } \left\{f\left(\sigma_{i j}, \beta_{i}\right)^{p^{k}}\right.\right.
$$

$$
\left.\left.-f\left(\sigma_{i j}, \beta_{i}\right)^{p^{k-1}} f\left(\delta_{i}, \beta_{i}\right)^{p^{k-1}(p-1)}\right\} D\left(\sigma_{i j}, 0\right)\right) \text {, }
$$


where $q_{0}=n_{0}$ and $q_{i}=n_{i}-1$ for $i=1, \ldots, m$. The restricted algebra $R(£(G, \delta, f))$ is therefore contained within the span of $R \cup T$. In the following discussion we will show that a basis for $R(\&(G, \delta, f))$ is $R \cup T \backslash$ ad $\left.u_{-\delta}\right\}$ when $G_{0} \neq\{0\}$ and $R \cup T \backslash\left\{\operatorname{ad} u_{-\delta}, D(\delta, 0)\right\}$ when $G_{0}=\{0\}$. It follows that $\operatorname{dim} R(\varrho(G, \delta, f))=\operatorname{dim} \mathscr{L}(G, \delta, f)+n-2 m$.

Definition. The column rank over $\Phi_{p}$ of a matrix $A$ with entries from $\Phi$ is the dimension of the vector space over $\Phi_{p}$ spanned by the columns of $A$. Denote this dimension by col $\operatorname{rank}_{\Phi_{p}}(A)$.

Lemma 2. If $G=G_{0}$, then col rank $\Phi_{p}\left(f\left(\sigma_{0 i}, \sigma_{0 j}\right)^{p}\right)=n$, the dimension of $G$ over $\Phi_{p}$.

Proof. Suppose col rank $\Phi_{p}\left(f\left(\sigma_{0 i}, \sigma_{0 j}\right)^{p}\right)<n$, that is, suppose that there exist elements $a_{1}, a_{2}, \ldots, a_{n} \in \Phi_{p}$, not all zero, such that

$$
\sum_{j=1}^{n} a_{j} f\left(\sigma_{0 i}, \sigma_{0 j}\right)^{p}=0
$$

for $i=1, \ldots, n$. Then from the biadditivity of $i$ we conclude that $f\left(\sigma_{0 i}, \Sigma_{j=1}^{n} a_{j} \sigma_{0 j}\right)^{p}=0$, or $f\left(\sigma_{0 i}, \Sigma_{j=1}^{n} a_{j} \sigma_{0 j}\right)=0$ for $i=1, \ldots, n$. This contradicts the nondegeneracy of $f$, whence the lemma is proved.

Lemma 3. Suppose $G=G_{1}$. Let $\left\{\beta_{1}, \ldots, \beta_{k}, \delta\right\}$ be a basis for $G$ where $f\left(\beta_{1}, \delta\right) \neq 0$, and let

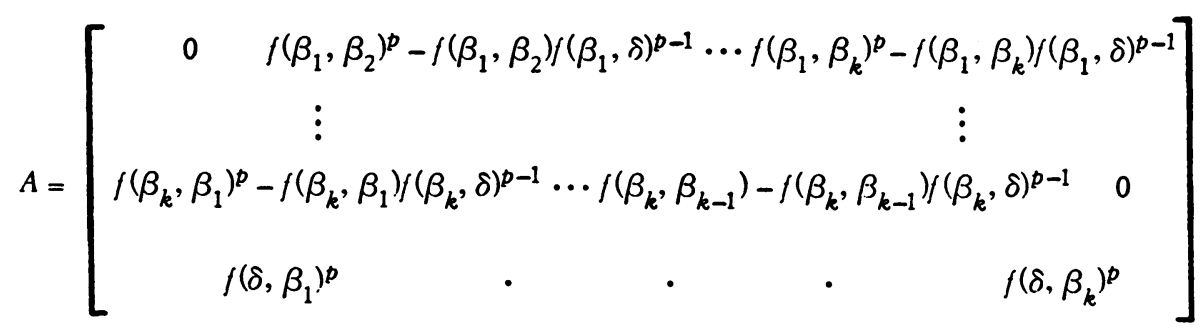

Then $\operatorname{col} \operatorname{rank}_{\phi_{p}}(A)=k$.

Proof. Suppose col $\operatorname{rank}_{\Phi_{p}}(A)<k$. Then there exist $a_{1}, \ldots, a_{k} \in \Phi_{p}$, not all zero, such that

$$
\sum_{j=1}^{k} a_{j}\left\{f\left(\beta_{i}, \beta_{j}\right)^{p}-f\left(\beta_{i}, \beta_{j}\right) f\left(\beta_{i}, \delta\right)^{p-1}\right\}=0
$$

for $i=1, \ldots, k$ and $\Sigma_{j=1}^{k} a_{j} f\left(\delta, \beta_{j}\right)^{p}=0$. For each of the first $k$ equalities we have 


$$
f\left(\beta_{i}, \sum_{j=1}^{k} a_{j} \beta_{j}\right)^{p}=f\left(\beta_{i}, \sum_{j=1}^{k} a_{j} \beta_{j}\right) f\left(\beta_{i}, \delta\right)^{p-1}
$$

or

$$
\left(f\left(\beta_{i}, \sum_{j=1}^{k} a_{j} \beta_{j}\right) / f\left(\beta_{i}, \delta\right)\right)^{p-1}=1,
$$

if $f\left(\beta_{i}, \delta\right) \neq 0$. Thus, for each $i=1, \ldots, k$, there exists $c_{i} \in \Phi_{p}$ such that $f\left(\beta_{i}, \sum_{j=1}^{k} a_{j} \beta_{j}\right)=c_{i} f\left(\beta_{i}, \delta\right)$ (if $f\left(\beta_{i}, \delta\right)=0$, then $\left.c_{i}=0\right)$. Now define $g: G \rightarrow \Phi$ and $h: G \rightarrow \Phi$ by $g(\alpha)=f(\alpha, \delta)$ and $h(\alpha)=f\left(\beta_{1}, \alpha\right)\left[f\left(\beta_{1}, \delta\right)\right]^{-1}$, whence $f(\alpha, \beta)=g(\alpha) h(\beta)-g(\beta) h(\alpha)$. Now

$$
\begin{aligned}
h\left(\sum_{j=1}^{k} a_{j} \beta_{j}-c_{1} \delta\right) & =f\left(\beta_{1}, \sum_{j=1}^{k} a_{j} \beta_{j}\right)\left[f\left(\beta_{1}, \delta\right)\right]^{-1}-f\left(\beta_{1}, c_{1} \delta\right)\left[f\left(\beta_{1}, \delta\right)\right]^{-1} \\
& =\left\{f\left(\beta_{1}, \sum_{j=1}^{k} a_{j} \beta_{j}\right)-c_{1} f\left(\beta_{1}, \delta\right)\right\}\left[f\left(\beta_{1}, \delta\right)\right]^{-1}=0
\end{aligned}
$$

and

$$
g\left(\sum_{j=1}^{k} a_{j} \beta_{j}-c_{1} \delta\right)=f\left(\sum_{j=1}^{k} a_{j} \beta_{j}-c_{1} \delta, \delta\right)=\sum_{j=1}^{k} a_{j} f\left(\beta_{j}, \delta\right) .
$$

But $\Sigma a_{j} f\left(\delta, \beta_{j}\right)^{p}=0$, so $g\left(\sum_{j=1}^{k} a_{j} \beta_{j}-c_{1} \delta\right)=0$. This implies the contradiction $f\left(\alpha, \sum_{j=1}^{k} a_{j} \beta_{j}-c_{1} \delta\right)=0$ for all $\alpha$, implying that col $\operatorname{rank}_{\Phi_{p}}(A)=k$.

Now suppose $G$ is arbitrary and $\left\{\sigma_{01}, \ldots, \sigma_{0 n_{0}}, \sigma_{11}, \ldots, \sigma_{1, n_{1}-1}, \delta_{1}\right.$, $\left.\ldots, \sigma_{m 1}, \ldots, \delta_{m}\right\}$ is a basis of $G$ over $\Phi_{p}$. Equation (1) shows that $\Re(\mathcal{R}(G, \delta, f)) \subseteq\left\langle D\left(\sigma_{i j}, 0\right)\right.$, ad $\left.x \mid x \in \mathcal{L}(G, \delta, f)\right\rangle$ and for the special case $k=1$ we have a matrix equation of the form:

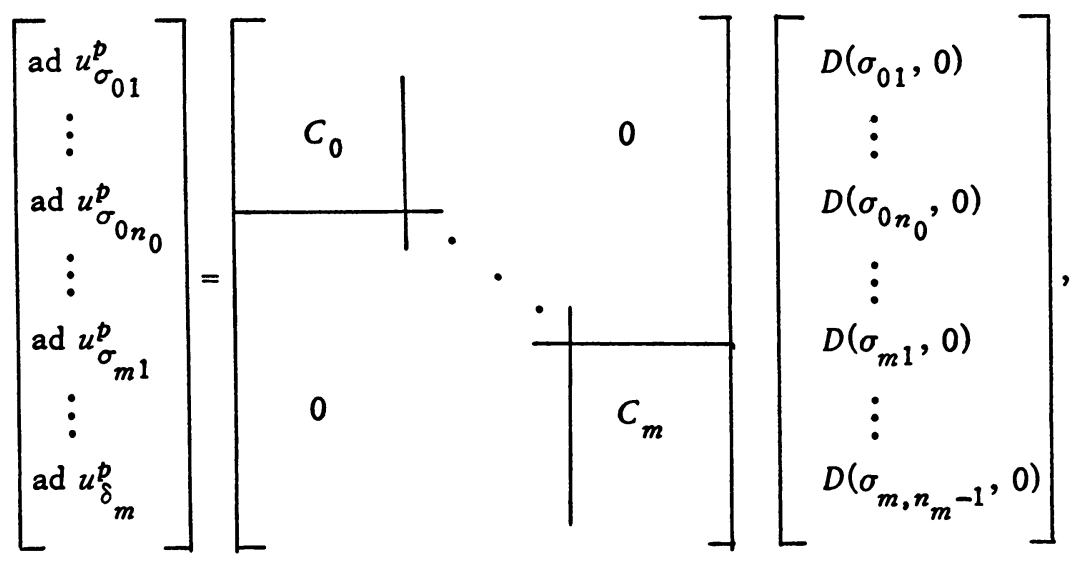


where $C_{0}=\left(f\left(\sigma_{0 i}, \sigma_{0 j}\right)^{p}\right)$ and, for $i>0, C_{i}$ is an $n_{i} \times\left(n_{i}-1\right)$ matrix of the form of the matrix in Lemma 3. Denote this matrix by $C$. To determine the coefficient matrix of the $D\left(\sigma_{i j}, 0\right)$ 's for higher powers of $p$, one merely raises the elements in $C$ to the appropriate pth power.

Lemma 4. Let $A=\left(a_{i j}\right)$ be an $r \times s$ matrix over a field $\Phi$ of characteristic $p>0$ and let $A_{p^{t}}=\left(a_{i j}^{p^{t}}\right)$ for $t \geq 0$. If col rank $\Phi_{p} A=s$, then $\operatorname{rank}_{\Phi}\left(A A_{p} \cdots A_{p}\right)^{T}=s$ for sufficiently large $t\left({ }^{T}\right.$ denotes transpose).

Proof. Since $\operatorname{rank}_{\Phi}\left(A \cdots A_{p}\right)^{T} \leq \operatorname{rank}_{\Phi}\left(A \cdots A_{p^{i+1}}\right)^{T} \leq s$ for all $i$ there exists some $t$ such that $\operatorname{rank}_{\Phi}\left(A \cdots A_{p t}\right)^{T}=\operatorname{rank}_{\Phi}\left(A \cdots A_{p t+1}\right)^{T}$. If $\operatorname{rank}_{\Phi}\left(A \cdots A_{p t}\right)^{T}<s$, then there exist $b_{1}, \ldots, b_{s} \in \Phi$, not all zero, such that $\sum_{j=1}^{s} b_{j} a_{i j}^{p^{v}}=0$ for all $i, 1 \leq i \leq r$, and all $v, 0 \leq v \leq t$. Note that this, and the choice of $t$, implies $\Sigma_{i=1}^{s} b_{j}^{a}{ }_{i j}^{t+1}=0$. Assume that the $b^{\prime}$ 's have been chosen so that the number of nonzero $b_{j}$ is minimal. In addition, assume $b_{1}=1$. Then

$$
0=\left(\sum_{j=1}^{s} b_{j} a_{i j}^{p^{\nu}}\right)^{p}=\sum_{j=1}^{s} b_{j}^{p} a_{i j}^{p^{v+1}}
$$

On the other hand, since $\sum_{j=1}^{s} b_{j} a_{i j}^{p+1}=0$ for $0 \leq v \leq t$ we have

$$
\sum_{j=1}^{s}\left(b_{j}^{p}-b_{j}\right) a_{i j}^{p^{v+1}}=0 \text {. }
$$

Extracting pth roots and using the minimality of the $b^{\prime} s$ (recall $b_{1}=1$ ) gives $b_{j}^{p}-b_{j}=0$ for all $j$, that is, $b_{j} \in \Phi_{p}$ for all $j$. This contradicts the assumption that col rank $\boldsymbol{\Phi}_{p} A=s$.

Returning to $C$, recall that the nondegeneracy of $f$ guarantees that col rank $\Phi_{p} C_{0}=n_{0}$ and col rank $\Phi_{p} C_{i}=n_{i}-1$ for $i>0$. Lemma 4 then allows us to conclude that

$$
\left\langle D\left(\sigma_{i j}, 0\right), \text { ad } x \mid x \in \mathfrak{Q}(G, \delta, f)\right\rangle \subseteq \mathfrak{R}(\mathfrak{Q}(G, \delta, f)) .
$$

Inclusion in the other direction was illustrated earlier, completing the proof of the main theorem.

Theorem. Let $\mathcal{L}(G, \delta, f)$ be a simple Lie algebra of Block. Then $\operatorname{dim} \mathfrak{R}(\mathcal{L}(G, \delta, f))=\operatorname{dim} \mathfrak{Q}(G, \delta, f)+n-2 m$.

Corollary. Two algebras of Block of the same dimension, $\mathscr{L}(G, \delta, f)$ and $\stackrel{P}{\propto}\left(G^{\prime}, \delta^{\prime}, f^{\prime}\right)$, are isomorphic only if $m(G)=m\left(G^{\prime}\right)$.

From the preceding discussion it is evident that for $\mathcal{\varrho}(G, \delta, f)$ and $\mathcal{L}\left(G^{\prime}, \delta^{\prime}, f^{\prime}\right)$ of the same dimension isomorphism is not guaranteed by the 
equality $m(G)=m\left(G^{\prime}\right)$. This follows from the fact that $R(\&(G, \delta, f))$ need not be isomorphic to $R\left(\&\left(G^{\prime}, \delta^{\prime}, f^{\prime}\right)\right)$. For example, let $m(G)=m\left(G^{\prime}\right)=0$ and $n=4$. Suppose $\left\{\beta_{1}, \beta_{2}, \beta_{3}, \beta_{4}\right\}$ and $\left\{\beta_{1}^{\prime}, \beta_{2}^{\prime}, \beta_{3}^{\prime}, \beta_{4}^{\prime}\right\}$ are bases for $G$ and $G^{\prime}$, respectively, where the matrices $\left(f\left(\beta_{i}, \beta_{j}\right)\right)$ and $\left(f\left(\beta_{i}^{\prime}, \beta_{j}^{\prime}\right)\right)$ are

$$
\left[\begin{array}{rrrr}
0 & 1 & 0 & x \\
-1 & 0 & 0 & 0 \\
0 & 0 & 0 & 1 \\
-x & 0 & -1 & 0
\end{array}\right] \text { and }\left[\begin{array}{rrrr}
0 & x & 0 & 1 \\
-x & 0 & 1 & 0 \\
0 & -1 & 0 & -1 / x \\
-1 & 0 & 1 / x & 0
\end{array}\right] \text {, }
$$

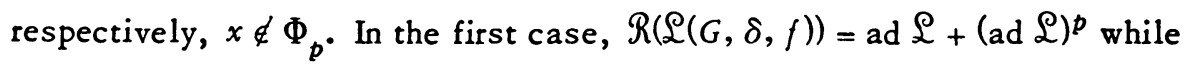
this is not true in the second.

\section{BIBLIOGR APHY}

1. Richard Block, New simple Lie algebras of prime characteristic, Trans. Amer. Math. Soc. 89 (1958), 421-449. MR 20 \#646.

DEPAR TMENT OF MATHEMATICS, UNIVERSITY OF OREGON, EUGENE, OREGON 97403 